\title{
ON THE DOMINANCE OF PARTIAL DIFFERENTIAL OPERATORS $\left({ }^{1}\right)$
}

\author{
BY \\ MARTIN SCHECHTER
}

1. Introduction. Let $G$ be a domain in Euclidean $n$-space and let $V$ be a set of functions defined on $G$. If $P_{1}, \cdots, P_{q}$ are partial differential operators, a fundamental problem in many investigations has been to determine those operators $R$ for which one has estimates of the form

$$
\|R u\|_{0} \leqq C \sum_{k=1}^{q}\left\|P_{k} u\right\|_{k}
$$

holding for all $u \in V$, where the \|\|$_{i}, i=0,1, \cdots, q$, are suitable norms. In fact, one can safely say that it is a rare investigation in partial differential equations which does not require estimates of the form (1.1). Of course, the validity of (1.1) depend upon all contributing factors $-G$, the $P_{k}, R, V$ as well as the norms. In many instances the choice of these factors is by no means clear for the particular problem at hand and must be left to the ingenuity of the investigator.

When (1.1) holds we say that $R$ is dominated by the $P_{k}$ over the set $V$ with respect to the given norms. The general problem, given $G, V$, the $P_{k}$ and the norms, is to determine all operators $R$ which are so dominated. This problem has not been solved in general even for some simple situations. However, many important results are known. The most popular norms are the maximum, $L^{p}$, and Hölder norms, with or without weight functions. Usually $V$ is a space of functions which satisfy given conditions at the boundary. When employing $L^{2}$ norms, the righthand side can be generalized to quadratic forms. There are results for systems as well.

If $V$ is determined by equations of the form

$$
Q_{j} u=0, \quad j=1,2, \cdots, q_{1},
$$

holding on the boundary $\partial G$ of $G$, one can formulate the problem in a slightly more general framework, namely to determine all $R$ for which

$$
\|R u\|_{0} \leqq C\left(\Sigma\left\|P_{k} u\right\|_{k}+\Sigma\left\|\mid Q_{j} u\right\| \|_{j}\right)
$$

Received by the editors October 2, 1961 and, in revised form, June 6, 1962.

(1) This paper represents results obtained under the sponsorship of the U. S. Atomic Energy Commission Contract AT (30-1)-1480. 
holds for all functions $u$, where the $\left|\|\mid\|_{j}\right.$ are suitable boundary norms. Of course, (1.3) will imply (1.1) for $u \in V$.

It would be extremely difficult to mention all known results for problems of this sort. Since we shall be primarily concerned with the $L^{2}$ norm we mention some recent investigations for that norm (without weight functions).

Aronszajn [3] investigated the case when $G$ is bounded and $P_{q}=1$. If $m^{\prime}$ is the maximum order of the $P_{k}$ and the $P_{k}$ dominate all operators of order $\leqq m^{\prime}$ on $V$, he calls the $P_{k}$ "coercive" over $V$. He determined necessary and sufficient conditions $\left({ }^{2}\right)$ for the $P_{k}$ to be coercive over $C^{m^{\prime}}(\bar{G})$. For the case $q=2$ and $P_{1}$ a strongly elliptic operator, Guseva [7], Nirenberg [10], and Browder [4] showed that $P_{1}$ is coercive over the set of those $u \in C^{m^{\prime}}(\bar{G})$ which have zero Dirichlet data on the boundary. In $[4 ; 10]$ an inequality due to Gåding [6] was employed. Later, this was generalized to an arbitrary elliptic operator $P_{1}$ satisfying a "root" condition by Schechter [11]. In addition, more general spaces $V$ defined by the equations of the form (1.2) where the $Q_{j}$ are differential operators were considered by Agmon-Douglis-Nirenberg [2] and Browder [5]. The case $q>2$ was also generalized by Agmon [1] and Schechter [12] to include the more general space $V$.

When $G$ is bounded, $q=1, P_{1}$ has constant coefficients, and $V$ is the set $C_{0}^{m^{\prime}}(G)$ of functions $u \in C^{m^{\prime}}(\bar{G})$ with compact support in $G$, Malgrange [9] and Hörmander [8] showed that $P_{1}$ always dominates $R=1$. When $R$ is dominated by $P_{1}$ over $C_{0}^{m^{\prime}}(G)$, Hörmander [8] calls it "weaker" than $P_{1}$. He gives a criterion for determining all operators $R$ which are weaker than a given $P_{1}\left({ }^{3}\right)$. Thomée [14] has treated the case when $R=1$ and $V$ is more general. He assumes that $P_{1}$ is homogeneous and that $G$ has a portion of its boundary situated in a hyperplane. $V$ was taken to be the set of functions which satisfy zero Dirichlet-type data on the planar part of the boundary and which vanish near the remaining portion. When $n=2$ and $P_{1}$ has simple (complex) characteristics, he also considered more general boundary conditions of the type (1.3). He calls the boundary condition "locally cogent" if $P_{1}$ dominates $R=1$.

In this paper we consider $G$ with part $\partial_{1} G$ of its boundary situated in a hyperplane. We consider functions $u \in C_{0}^{m^{\prime}}\left(G \cup \partial_{1} G\right)$, i.e., function in $C^{m^{\prime}}(\bar{G})$ which vanish near $\partial G-\partial_{1} G$ and outside compact sets. $R$ and the $P_{k}$ are homogeneous, constant coefficient operators of orders $m, m_{k}$, respectively, $k=1,2, \cdots, q$. The boundary conditions are expressed in the form (1.2) where the $Q_{j}$ are also homogeneous, constant coefficient operators. We employ norms slightly more general than the $L^{2}(G)$ norm.

Our general results are stated in $\S \S 2$ and 6 . As a special case they imply sufficient conditions for an inequality of the form

(2) We do not give the regularity assumptions made for some of the reported results.

(3) His methods carry over immediately to the case $q>1$, as long as the $P_{k}$ have constant coefficients. 


$$
\|R u\| \leqq C\left(\sum_{k}\left\|P_{k} u\right\|+\sum_{j}\left\|Q_{j} u\right\| \|_{j}\right)
$$

to hold for all $u \in C_{0}^{\infty}\left(G \cup \partial_{1} G\right)$, where \|\| is the $L^{2}(G)$ norm and the \|\|$\|_{j}$ are suitable boundary norms. To give an idea as to their nature, we describe one theorem for $n=2$. It is simpler to state and indicates what one can expect for $n>2$.

Assume that each $m_{k} \geqq m$. We consider the characteristic polynomials $P_{k}(\xi, \eta)$ of the $P_{k}$, where $\eta$ corresponds to the coordinate normal to the plane portion of $\partial G$. The multiplicities of the real $\eta$ roots of $P_{k}(\xi, \eta)$ are decreased by $m_{k}-m$ and we consider the greatest common divisor $P(\xi, \eta)$ of the resulting polynomials. We let $P^{+}(1, \eta)$ (resp. $\left.P^{-}(1, \eta)\right)$ be the largest divisor of $P(1, \eta)$ having only roots with non-negative (resp. nonpositive) imaginary parts and denote its degree by $\mathrm{m}^{+}$(resp. $\mathrm{m}^{-}$). Then our theorem states that there is an inequality of the form (1.4) holding for all $u \in C_{0}^{m^{\prime}}\left(G \cup \partial_{1} G\right)$ if $R$ is weaker than the $P_{k}$ and there are $m^{+}$(resp. $m^{-}$) of the polynomials $Q_{j}(1, \eta)$ which are of degree less than $m^{\prime}$ and are linearly independent modulo $P^{+}(1, \eta)\left(\right.$ resp. $\left.P^{-}(1, \eta)\right)$. More precise statements can be found in $\$ 2$.

We wish to thank S. Agmon and M. Herschorn for interesting discussions.

2. Definitions and partial results. Let $E^{n}$ denote Euclidean $n$-space with generic points $x=\left(x_{1}, \cdots, x_{n}\right)$. Let $G$ be a domain in the halfspace $x_{n} \geqq 0$ with a portion $\partial_{1} G$ of its boundary $\partial G$ contained in the hyperplane $x_{n}=0$. For any integer $m \geqq 1$ we consider linear homogeneous partial differential operators of order $m$ with constant coefficients. We write them in the form

$$
P=\sum_{|\mu|+s=m} a_{\mu s} D^{\mu} D_{n}^{s},
$$

where $\mu=\left(\mu_{1}, \cdots, \mu_{n-1}\right)$ is an $n-1$ dimensional multi-index of non-negative integers, $|\mu|=\mu_{1}+\cdots+\mu_{n-1}$ the $a_{\mu s}$ are complex constants,

$$
D^{\mu}=(-i)^{|\mu|} \partial^{|\mu|} / \partial x_{1}^{\mu_{1}} \cdots \partial x_{n-1}^{n-1},
$$

and

$$
D_{n}^{s}=(-i)^{s} \partial^{s} / \partial x_{n}^{s} .
$$

We shall consider the class $C_{0}^{\infty}\left(G \cup \partial_{1} G\right)$ of complex valued, infinitely differentiable functions defined on the closure $\bar{G}$ of $G$ which have compact support in $G \cup \partial_{1} G$, i.e., which vanish near $\partial G-\partial_{1} G$ and outside compact sets. The characteristic polynomial corresponding to $P$ is

$$
P(\xi, \eta)=\sum_{|\mu|+s=m} a_{\mu s} \xi^{\mu} \eta^{s},
$$


where $\xi=\left(\xi_{1}, \cdots, \xi_{n-1}\right)$ is an $n-1$ dimensional vector and

$$
\xi^{\mu}=\xi_{1}^{\mu_{1}} \cdots \xi_{n-1}^{\mu_{n-1}}
$$

Let $P_{k}, Q_{j}$ be homogeneous, constant coefficient operators of orders $m_{k}, v_{j}$, respectively, $k=1,2, \cdots, n_{1} ; j=1,2, \cdots, n_{2}$. Let

$$
P_{k}(\xi, \eta)=\sum_{|\mu|+s=m_{k}} a_{k \mu s} \xi^{\mu} \eta^{s}, \quad k=1,2, \cdots, n_{1},
$$

and

$$
Q_{j}(\xi, \eta)=\sum_{|\mu|+s=v_{j}} b_{k \mu s} \xi^{\mu} \eta^{s}, \quad j=1,2, \cdots, n_{2},
$$

be the corresponding characteristic polynomials. For each $\xi$ such that $|\xi|^{2} \equiv \xi_{1}^{2}+\cdots+\xi_{n-1}^{2}=1$, let $P_{\xi}(\eta)$ be the greatest common divisor of the $P_{k}(\xi, \eta)$, and let $P_{\xi}^{+}(\eta)$ be the largest factor of $P_{\xi}(\eta)$ having only roots with nonnegative imaginary parts. Denote the degree of $P_{\xi}^{+}(\eta)$ by $m^{+}(\xi)$.

For a function $u \in C_{0}^{\infty}\left(G \cup \partial_{1} G\right)$ we shall consider norms defined by means of Fourier transforms. We consider functions in $C_{0}^{\infty}\left(G \cup \partial_{1} G\right)$ to be defined as zero outside $\bar{G}$ and $\operatorname{set}\left({ }^{4}\right)$

$$
\begin{aligned}
\tilde{u}(\xi, \eta) & =\int e^{-i\left(\xi x^{\prime}+\eta y\right)} u\left(x^{\prime}, y\right) d x^{\prime} d y, \\
\hat{u}(\xi) & =i \int e^{-i \xi x^{\prime}} u\left(x^{\prime}, 0\right) d x^{\prime},
\end{aligned}
$$

where $x^{\prime}=\left(x_{1}, \cdots, x_{n-1}\right), y=x_{n}$, and $\xi x^{\prime}=\xi_{1} x_{1}+\cdots+\xi_{n-1} x_{n-1}$. For any real number $r$ we define

$$
\begin{aligned}
|u|_{r}^{2} & =\int|\xi|^{2 r}|\tilde{u}|^{2} d \xi d \eta, \\
\langle u\rangle_{r}^{2} & =\int|\xi|^{2 r}|\hat{u}|^{2} d \xi .
\end{aligned}
$$

By Parseval's identity, $|u|_{0}$ is equivalent to the $L^{2}(G)$ norm of $u$.

THEOREM 2.1. Let $R$ be a homogeneous constant coefficient operator of order $m$ with characteristic polynomial $R(\xi, \eta)$. Then there is a constant $C$ such that

$$
|R u|_{r} \leqq C\left(\sum_{k}\left|P_{k} u\right|_{r+m-m_{k}}+\sum_{j}\left\langle Q_{j} u\right\rangle_{r+m-v_{j}-1 / 2}\right)
$$

for all $u \in C_{0}^{\infty}\left(G \cup \partial_{1} G\right)$ and real numbers $r$, if

(a) there is an integer $m_{0} \geqq 0$ such that $a_{k \mu s}=0$ for $s>m_{0}$ and all $k, \mu$, while

for $|\xi|=1$;

$$
\sum_{k}\left|\sum_{\mu} a_{k \mu m} \xi^{\mu}\right|: \neq 0
$$

(4) When limits of integration are not indicated, integration is to be taken over the whole space considered. 
(b) there is a constant $K_{0}$ such that

$$
|R(\xi, \eta)|^{2} \leqq K_{0} \Sigma|\xi|^{2\left(m-m_{k}\right)}\left|P_{k}(\xi, \eta)\right|^{2}
$$

for all real $\xi, \eta, \xi \neq 0$;

(c) the $Q_{j}(\xi, \eta)$ cover the $P_{k}(\xi, \eta)$. By this we mean that for each $\xi$ such that $|\xi|=1$ there are $m^{+}(\xi)$ of the $Q_{j}(\xi, \eta)$ which are of degree less than $m_{0}$ in $\eta$ and are linearly independent modulo $P_{\xi}^{+}(\eta)$.

We have so far made no restriction on $G$ other than that it be contained in the halfspace $x_{n} \geqq 0$. We now assume that it is bounded. Our general results for this case (Theorems 6.1 and 6.2) are too complicated to state here. We content ourselves with considering special cases.

Let $P(\xi, \eta)$ be the characteristic polynomial corresponding to a constant coefficient partial differential operator $P$. At each point $\xi$ of $|\xi|=1$ we can factor $P(\xi, \eta)$ in the form

$$
P(\xi, \eta)=a(\xi) \prod_{t=0}^{N(\xi)}\left(\eta-\lambda_{t}(\xi)\right)^{s_{t}(\xi)},
$$

where $a(\xi) \neq 0, N(\xi)$, and $s_{t}(\xi)$ are, in general, discontinuous functions of $\xi$. The $\lambda_{t}(\xi)$ are considered roots of $P(\xi, \eta)$ with multiplicities $s_{t}(\xi)$, respectively. For any integer $q$ we set

$$
s_{t}^{q}\left(\xi_{0}\right)=\max \left[s_{t}\left(\xi_{0}\right)-q, 0\right]
$$

if $\lambda_{t}\left(\xi_{0}\right)$ is real and $\operatorname{Im} \lambda_{t}(\xi) \leqq 0$ in a neighborhood of $\xi_{0}$. Otherwise we set

$$
s_{t}^{q}\left(\xi_{0}\right)=s_{t}\left(\xi_{0}\right)
$$

We define

$$
P^{(q)}(\xi, \eta) \equiv a(\xi) \prod\left(\eta-\lambda_{t}(\xi)^{s_{t}^{q}(\xi)}\right.
$$

when $q \geqq 0$, and $P^{(q)}(\xi, \eta)=0$ otherwise. We say that the polynomials $P_{k}(\xi, \eta)$ are collectively normal if they satisfy hypothesis (a) of Theorem 2.1.

THEOREM 2.2. For any set of non-negative integers $\rho,\left\{l_{k}\right\}_{k=1}^{n_{1}}$, satisfying $\rho \leqq \max l_{k}$ and any real $r \geqq \max _{j, k}\left(m_{k}, v_{j}+1\right)$ there is a constant $C$ such that

$$
|R u|_{r-m} \leqq C\left\{\sum_{k}\left|P_{k} u\right|_{r-m_{k}+l_{k}}+\sum_{j}\left\langle Q_{j} u\right\rangle_{r-v+\rho}\right\}
$$

for all $u \in C_{0}^{\infty}\left(G \cup \partial_{1} G\right)$, if

(a) the polynomials $P_{k}(\xi, \eta)$ are collectively normal;

(b) there are non-negative integers $\rho_{k} \leqq \min \left(\rho+1, l_{k}\right)$ such that 


$$
|R(\xi, \eta)|^{2} \leqq K \sum_{k} \sum_{s=0}^{\rho_{k}}|\xi|^{2\left(m-m_{k}+s\right)}\left|\frac{\partial^{s} P_{k}(\xi, \eta)}{\partial \eta^{s}}\right|^{2}
$$

for all real $\xi, \eta, \xi \neq 0$;

(c) the $Q_{j}(\xi, \eta)$ cover the polynomials $P_{k}^{\left(\sigma_{k}\right)}(\xi, \eta)$, where

$$
\sigma_{k}=\min \left(l_{k}-\rho_{k}, l_{k}-\rho\right) \text {. }
$$

THEOREM 2.3. Inequality (2.7) holds for any operator $R$ of order $m$ if hypotheses (a)-(c) of Theorem 2.2 are replaced by the following:

(a') the polynomials $P_{k}(\xi, \eta)$ are collectively normal and $m_{0} \geqq m$;

(b') for no $\xi$ of $|\xi|=1$ does $P_{\xi}(\eta)$ have any real roots with multiplicities greater than $\min \left(\rho+1, \min l_{k}\right)$;

(c') the $Q_{j}(\xi, \eta)$ cover the polynomials $P_{k}^{\left(l_{k}-\rho\right)}(\xi, \eta)$.

We end this section by stating some theorems in two dimensions. They are slightly stronger than Theorems 2.2 and 2.3 and indicate the power of our general results (Theorems 6.1 and 6.2). We now assume that $n=2$. Hence $\xi=\xi_{1}$, a number. The only restriction we make on $G$ is that it be contained in a strip $0 \leqq x_{n} \leqq d$.

THEOREM 2.4. For any set of non-negative numbers $\left\{l_{k}\right\}$ there is a constant $C$ such that

$$
|R u|_{r-m} \leqq C\left\{\sum_{k}\left|P_{k} u\right|_{r-m_{k}+l_{k}}+\sum_{j} \sum_{s=0}^{1}\left\langle Q_{j} u\right\rangle_{r-v_{j}+((s-1) / 2)}\right\}
$$

for all real $r$ and $u \in C_{0}^{\infty}\left(G \cup \partial_{1} G\right)$, if

(a) there is a constant $K_{1}$ such that

$$
|R(\xi, \eta)|^{2} \leqq K_{1} \sum_{k}|\xi|^{2\left(m-m_{k}+l_{k}\right)} \sum_{s+t=l_{k}}\left|\frac{\partial^{s+t} P_{k}(\xi, \eta)}{\partial \xi s \partial \eta^{t}}\right|^{2}
$$

for all real $\xi, \eta, \xi \neq 0$;

(b) the $Q_{j}(\xi, \eta)$ cover the polynomials $P_{k}^{\left(l_{k}\right)}(\xi, \eta)$.

A special case of Theorem 2.4 can be put in a convenient form if we employ the following concept due to Hörmander [8]. The polynomial $R(\xi, \eta)$ is said to be weaker than the polynomials $P_{k}(\Sigma, \eta)$ if there is a constant $C$ such that

$$
|R(\xi, \eta)|^{2} \leqq C \sum\left|\frac{\partial^{s+t} P_{k}(\xi, \eta)}{\partial \xi^{s} \partial \eta^{t}}\right|^{2}
$$

for all real $\xi, \eta$, where summation is taken over all non-negative integers $s$ and $t$ as well as all polynomials $P_{k}(\xi, \eta)$. By Hörmander's result [8], this is a necessary and sufficient condition for

$$
|R \phi|_{0} \leqq C \sum\left|P_{k} \phi\right|_{0}
$$

to hold for $\phi \in C_{0}^{\infty}(G)$. When each $m_{k} \geqq m$ we have 
THEOREM 2.5. There is a constant $C$ such that

$$
|R u|_{\tau} \leqq C\left(\sum_{k}\left|P_{k} u\right|_{\tau}+\sum_{j} \sum_{s=0}^{1}\left\langle Q_{j} u\right\rangle_{\tau+m-v_{j}+((s-1) / 2)}\right)
$$

for all $u \in C_{0}^{\infty}\left(G \cup \partial_{1} G\right)$ and real $\tau$ if

(a) $R(\xi, \eta)$ is weaker than the $P_{k}(\xi, \eta)$;

(b) the $Q_{j}(\xi, \eta)$ cover the $P_{k}^{\left(m_{k}-m\right)}(\xi, \eta)$.

The proof of Theorem 2.1 will be given in $\S 4$. It is based on the results of $\S 3$. The proofs of Theorems 2.2-2.5 will be given in $\S 6$ together with more general statements and theorems. The method of proof is developed in $\$ 5$.

3. Unbounded domains in halfspace. In this section we allow $G$ to be unbounded. For any integer $m \geqq 1$ we consider polynomials in $\eta$ of the form

$$
P(\xi, \eta)=\sum_{s=0}^{m} a_{s}(\xi) \eta^{s}
$$

where each coefficient $a_{s}(\xi)$ is an analytic function of $\xi$ on some closed subset $\Xi$ of the unit sphere $|\xi|=1$ and is homogeneous in $\xi$ of degree $m-s$. Such a polynomial will be called a characteristic polynomial of order $m$. Its normal order is the highest index $h$ for which $a_{h}(\xi) \not \equiv 0$ in $\Xi$. Thus any characteristic polynomial $P(\xi, \eta)$ can be written in the form

$$
P(\xi, \eta)=a_{h}(\xi) \prod_{t=1}^{h}\left(\eta-\lambda_{t}(\xi)\right)
$$

where each root $\lambda_{t}(\xi)$ is homogeneous of degree one. Moreover, it is well known that when $a_{h}(\xi) \neq 0, \Xi$ can be decomposed into a finite number of sets on each of which the $\lambda_{t}(\xi)$ are continuous, single valued functions (cf., e.g., $[15$, p. 126]).

As before, we consider a function $u \in C_{0}^{\infty}\left(G \cup \partial_{1} G\right)$ to be defined as a zero outside $\bar{G}$ and let

$$
\tilde{u}(\xi, \eta)=\int e^{-i\left(\xi x^{\prime}+\eta y\right)} u\left(x^{\prime}, y\right) d x^{\prime} d y
$$

denote its Fourier transform, where $x^{\prime}=\left(x_{1}, x_{2}, \cdots, x_{n-1}\right), y=x_{n}$, and $\xi x^{\prime}=\xi_{1} x_{1}+\cdots+\xi_{n-1} x_{n-1}$. We also consider the "tangential" Fourier transform

and set

$$
\hat{u}(\xi)=i \int e^{-i \xi x^{\prime}} u\left(x^{\prime}, 0\right) d x^{\prime}
$$

$$
\begin{aligned}
\omega_{s} & =|\xi|^{-s-1}\left(D_{n}^{s} u \hat{)} ; \quad \Omega_{s}=\sum_{t=0}^{s-1} \eta^{s-t-1}\left(D_{n}^{t} u\right)^{\hat{\gamma}}, \quad s=0,1,2, \cdots\right. \\
l u & =\left(\hat{u},\left(D_{n} u\right)^{\hat{\gamma}}, \cdots,\left(D_{n}^{j} u\right)^{\hat{\vartheta}}, \cdots\right) \\
L u & =\left(\Omega_{0}, \Omega_{1}, \cdots, \Omega_{j}, \cdots\right)
\end{aligned}
$$


Corresponding to each characteristic polynomial $P(\xi, \eta)$ we define the linear operators

$$
\begin{aligned}
& (P u)^{\sim}=P(\xi, \eta) \tilde{u}+P(\xi, L u), \\
& (P u)^{\hat{y}}=P(\xi, l u)
\end{aligned}
$$

where, for any vector $w=\left(w_{0}, w_{1}, \cdots, w_{s}, \cdots\right)$ we employ the notation

$$
P(\xi, w)=\sum_{s=0}^{m} a_{s}(\xi) w_{s} .
$$

Let $R(\xi, \eta), P_{k}(\xi, \eta), Q_{j}(\xi, \eta)$ be characteristic polynomials of orders $m, m_{k}, v_{j}$, respectively, $k=1,2, \cdots, n_{1}, j=1,2, \cdots, n_{2}$, with coefficients defined on the same closed set $\Xi$ of $|\xi|=1$. At each point $\xi \in \Xi$ let $P_{\xi}(\eta)$ be the greatest common divisor of the $P_{k}(\xi, \eta)$ normalized by having its leading coefficient equal to one. We take $P_{\xi}^{+}(\eta)$ to be that factor of $P_{\xi}(\eta)$ containing only the roots of $P_{\xi}(\eta)$ with non-negative imaginary parts and having them with the same multiplicity as $P_{\xi}(\eta)$. Thus $P_{\xi}^{+}(\eta)$ has only roots with non-negative imaginary parts and $P_{\xi}(\eta) / P_{\xi}^{+}(\eta)$ has only roots with negative imaginary parts. Denote the normal order of $P_{\xi}^{+}(\eta)$ by $m^{+}(\xi)$.

If the maximum normal order of the $P_{k}(\xi, \eta)$ is $m_{0}$, we shall say that $\Xi$ has property A relative to the $P_{k}(\xi, \eta)$ if for at least one of them, say $P_{1}(\xi, \eta)$, the coefficient of $\eta^{m 0}$ does not vanish anywhere in $\Xi$ and the roots $\lambda_{t}(\xi)$ are single valued there.

THEOREM 3.1. There is a constant $C$ such that

$$
\begin{aligned}
& \int\left|(R u)^{\sim}\right|^{2} d \eta \\
& \quad \leqq C\left\{\sum_{k}|\xi|^{2\left(m-m_{k}\right.} \int\left|\left(P_{k} u\right)^{\sim}\right|^{2} d \eta+\sum_{j}|\xi|^{2\left(m-v_{j}\right)-1}\left|\left(Q_{j} u\right)^{\wedge}\right|^{2}\right\}
\end{aligned}
$$

for all $u \in C_{0}^{\infty}\left(G \cup \partial_{1} G\right)$ and $\xi$ such that $\xi /|\xi| \in \Xi$ if

(a) there is a constant $K_{0}$ such that

$$
|R(\xi, \eta)|^{2} \leqq K_{0} \sum_{k}|\xi|^{2\left(m-m_{k}\right)}\left|P_{k}(\xi, \eta)\right|^{2}
$$

for all $\xi \in \Xi$ and real $\eta$;

(b) $\Xi$ has property A relative to the $P_{k}(\xi, \eta)$; and

(c) for each $\xi \in \Xi$ the polynomials $Q_{j}(\xi, \eta)$ cover the $P_{k}(\xi, \eta)$. By this we mean that there are at least $m^{+}(\xi)$ of the $Q_{j}(\xi, \eta)$ which are of normal order less than $m_{0}$ and are linearly independent modulo $P_{\xi}^{+}(\eta)$.

The proof of Theorem 3.1 will be accomplished by means of the following lemmas. 
LEMMA 3.1. If the $t_{j}$ are real and the $E_{j}$ and $F_{j}$ complex, then

$$
\begin{aligned}
\left(\sum t_{j}\left|E_{j}\right|^{2}\right)\left(\sum t_{k}\left|F_{k}\right|^{2}\right)- & \left|\sum t_{j} E_{j} F_{j}\right|^{2} \\
& =\frac{1}{2} \sum t_{j} t_{k}\left|E_{j} F_{k}-E_{k} F_{j}\right|^{2} .
\end{aligned}
$$

Proof. Direct verification.

LeMma 3.2 .

$$
\begin{aligned}
\left(\sum\left|P_{k}\right|^{2}-\varepsilon|R|^{2}\right)\left(\sum\left|\left(P_{k} u\right)^{\sim}\right|^{2}\right. & \left.-\varepsilon\left|(R u)^{\sim}\right|^{2}\right)-\left|\sum \bar{P}_{k}\left(P_{k} u\right)^{\sim}-\varepsilon \bar{R}(R u)^{\sim}\right|^{2} \\
& =\frac{1}{2} \sum\left|P_{k} S_{j}-P_{j} S_{k}\right|^{2}-\frac{1}{2} \varepsilon \sum\left|P_{k} T-R S_{k}\right|^{2},
\end{aligned}
$$

where

$$
S_{k}=P_{k}(\xi, L u), \quad T=R(\xi, L u) .
$$

Proof. Set $t_{0}=-\varepsilon, E_{0}=R, F_{0}=(R u)^{\sim}, t_{k}=1, E_{k}=P_{k}, F_{k}=\left(P_{k} u\right)^{\sim}$, $k=1,2, \cdots, n_{1}$, in Lemma 3.1 .

COROllary 3.1.

$$
\begin{aligned}
\left(\sum\left|P_{k}\right|^{2}\right)\left(\Sigma\left|\left(P_{k} u\right)^{\sim}\right|^{2}\right)-\left|\sum \bar{P}_{k}\left(P_{k} u\right)^{\sim}\right|^{2} & \\
= & \frac{1}{2} \Sigma\left|P_{k} S_{j}-P_{j} S_{k}\right|^{2} .
\end{aligned}
$$

LEMMA 3.3. Let $H(\xi, \eta)$ be a characteristic polynomial and $\lambda$ any complex number. If $V=H(\xi, L u)$, then

$$
\left|\sum \bar{P}_{k}\left(P_{k} u\right)^{\sim}\right|^{2} \geqq 2 \sum\left|P_{k}\right|^{2} \operatorname{Re} \lambda(H u)^{\sim}+2 \operatorname{Re} \lambda \Sigma \bar{P}_{k}\left(H S_{k}-V P_{k}\right)-|\lambda H|^{2} .
$$

Proof.

$$
\begin{aligned}
\left|\Sigma \bar{P}_{k}\left(P_{k} u\right)^{\sim}\right|^{2} & =\left|\sum \bar{P}_{k}\left(P_{k} u\right)^{\sim}-\overline{\lambda H}\right|^{2}+2 \operatorname{Re} \lambda H \sum \bar{P}_{k}\left(P_{k} u\right)^{\sim}-|\lambda H|^{2} \\
& \geqq 2\left(\sum\left|P_{k}\right|^{2}\right) \operatorname{Re} \lambda H \tilde{u}+2 \operatorname{Re} \lambda H \sum \bar{P}_{k} S_{k}-|\lambda H|^{2} \\
& =2\left(\sum\left|P_{k}\right|^{2}\right) \operatorname{Re} \lambda(H u)^{\sim}+2 \operatorname{Re} \lambda \sum \bar{P}_{k}\left(H S_{k}-V P_{k}\right)-|\lambda H|^{2}
\end{aligned}
$$

LEMMA 3.4. For $u \in C_{0}^{\infty}\left(G \cup \partial_{1} G\right)$

$$
\int \tilde{u} d \eta=-\pi i \hat{u}
$$

where the integral is taken in the Cauchy principal value sense.

The proof of Lemma 3.4 was given in [12]. 
THEOREM 3.2. For $u \in C_{0}^{\infty}\left(G \cup \partial_{1} G\right)$

$$
\Sigma \int\left|P_{k} u\right|^{2} d \eta \geqq\left[\int \frac{|H|^{2}}{\sum\left|P_{k}\right|^{2}} d \eta\right]^{-1}\left|\int \frac{\sum \bar{P}_{k}\left(H S_{k}-P_{k} V\right)}{\sum\left|P_{k}\right|^{2}} d \eta-\pi i(H u)^{\wedge}\right|^{2} \text {. }
$$

Proof. Set

$$
J_{0}=\Sigma\left|P_{k}\right|^{2}
$$

Then by Lemma 3.3 for any complex $\lambda$

$$
J_{0}^{-1}\left|\Sigma \bar{P}_{k}(P u)^{\sim}\right|^{2} \geqq 2 \operatorname{Re} \lambda\left\{J_{0}^{-1} \sum \bar{P}_{k}\left(H S_{k}-P_{k} V\right)+(H u)^{\sim}\right\}-J_{0}^{-1}|\lambda H|^{2} .
$$

Integrating, we have by Lemma 3.4

$$
\begin{aligned}
\int J_{0}^{-1}\left|\Sigma \bar{P}_{k}\left(P_{k} u\right)^{\sim}\right|^{2} d \eta \geqq & 2 \operatorname{Re} \lambda\left\{\int J_{0}^{-1} \sum \bar{P}_{k}\left(H S_{k}-P_{k} V\right) d \eta-\pi i(H u)^{\wedge}\right\} \\
& -|\lambda|^{2} \int J_{0}^{-1}|H|^{2} d \eta
\end{aligned}
$$

Take

$$
\bar{\lambda}=\left[\int \frac{|H|^{2}}{J_{0}} d \eta\right]^{-1}\left\{\int \frac{\sum \bar{P}_{k}\left(H S_{k}-P_{k} V\right)}{J_{0}} d \eta-\pi i(H u)^{\wedge}\right\}
$$

Thus

$$
\int J_{0}^{-1}\left|\sum \bar{P}_{k}\left(P_{k} u\right)^{\sim}\right|^{2} d \eta \geqq\left[\int \frac{|H|^{2}}{J_{0}} d \eta\right]^{-1}\left|\int \frac{\sum \bar{P}_{k}\left(H S_{k}-P_{k} V\right)}{J_{0}} d \eta-\pi i(H u)^{\wedge}\right|^{2} \text {. }
$$

Since

$$
\Sigma\left|\left(P_{k} u\right)^{\sim}\right|^{2} \geqq J_{0}^{-1}\left|\Sigma \bar{P}_{k}\left(P_{k} u\right)^{\sim}\right|^{2}
$$

by Corollary 3.1, the result follows.

LEMMA 3.5. Let

$$
P(\xi, \eta)=\sum_{s=0}^{p_{1}} a_{s}(\xi) \eta^{s} ; \quad H(\xi, \eta)=\sum_{s=0}^{h_{1}} b_{s}(\xi) \eta^{s}
$$

be characteristic polynomials of normal orders $p$ and $h$, respectively, with $p \geqq h$. If $S=P(\xi, L u)$ and $V=H(\xi, L u)$, then $H S-P V$ is of degree $p-1$ in $\eta$ with leading coefficient equal to $a_{p}(H u)^{\wedge}-b_{p}(P u)^{\wedge}$.

Proof. Direct verification.

LEMMA 3.6. Let $M_{1}(z)$ be a polynomial of degree $m$ with leading coefficient $a$. Assume that there is a polynomial $M_{2}(z)$ of degree $m-1$ with leading coefficient $b$ and such that $M_{1}(z) / M_{2}(z)$ does not vanish for $\operatorname{Im} z \geqq 0$. Then

$$
\int_{-\infty}^{\infty} \frac{M_{2}(\tau)}{M_{1}(\tau)} d \tau=-\pi i \frac{a}{b},
$$

where the integral is taken in the Cauchy principal value sense. 
Proof. Contour integration.

LeMma 3.7. If the polynomials $\left\{Q_{j}(z)\right\}$ are linearly independent modulo a polynomial $P(z)$ and the polynomials $\left\{N_{t}(z)\right\}$ are linearly independent, then the set $\left\{Q_{j}(z), P(z) N_{t}(z)\right\}$ is linearly independent.

Proof. Obvious.

We are now ready for the

Proof of Theorem 3.1. Set $m^{\prime}=\max m_{k}$. From the statement of the theorem we see that we may assume that $R$ and the $P_{k}$ are each of order $m^{\prime}$ and that each $Q_{j}$ is of order $m^{\prime}-1$. Next put

$$
J_{\varepsilon}=\Sigma\left|P_{k}\right|^{2}-\varepsilon|R|^{2}=J_{0}-\varepsilon|R|^{2} .
$$

By $(3.2)\left(^{5}\right)$ if $0<\varepsilon<\frac{1}{2} K_{0}^{-1}$,

$$
2 J_{\varepsilon}=J_{0}+\left(J_{0}-K_{0}^{-1}|R|^{2}\right)+\left(K_{0}^{-1}-2 \varepsilon\right)|R|^{2} \geqq J_{0} .
$$

Thus by Lemma 3.2

$$
\begin{aligned}
\int\left(\Sigma\left|\left(P_{k} u\right)^{\sim}\right|^{2}-\varepsilon\left|(R u)^{\sim}\right|^{2}\right) d \eta & \geqq-\frac{\varepsilon}{2} \int J_{\varepsilon}^{-1} \Sigma\left|P_{k} T-R S_{k}\right|^{2} d \eta \\
& \geqq-\varepsilon \int J_{0}^{-1} \Sigma\left|P_{k} T-R S_{k}\right|^{2} d \eta
\end{aligned}
$$

We note that the last integral in (3.3) exists since $P T_{k}-R S_{k}$ is of degree less than $m_{0}$ in $\eta$ (Lemma 3.5).

Let $H_{1}(\xi, \eta), H_{2}(\xi, \eta), \cdots, H_{q}(\xi, \eta)$ be the set of all characteristic polynomials of the form

$$
|\xi|^{m^{\prime}-\sigma-l-1} \eta^{\sigma} \prod_{t=1}^{l}\left(\eta-\lambda_{t}(\xi)\right), \quad 0 \leqq l<m_{0}, \quad 0 \leqq \sigma<m_{0}-l,
$$

where the $\lambda_{t}(\xi)$ are chosen from the roots of $P_{1}(\xi, \eta)\left({ }^{6}\right)$. Thus the characteristic polynomials $H_{i}(\xi, \eta)$ are of order $m^{\prime}-1$ and normal order less than $m_{0}$. By Theorem 3.2,

where

$$
\begin{gathered}
\sum_{s=1}^{q} \alpha_{s}(\xi)\left|\int J_{0}^{-1} \sum_{k} \bar{P}_{k}\left(H_{s} S_{k}-P_{k} V_{s}\right) d \eta-\pi i\left(H_{s} u\right)^{\wedge}\right|^{2} \\
\leqq q \int \Sigma\left|\left(P_{k} u\right)^{\sim}\right|^{2} d \eta
\end{gathered}
$$

$$
\alpha_{s}(\xi)=\left[\int \frac{\left|H_{s}\right|^{2}}{J_{0}} d \eta\right]^{-1}
$$

(5) We note that by homogeneity, (3.2) holds for all real $\xi$ and $\eta$ such that $\xi /|\xi| \in \Xi$.

(6) Recall that we assume that the coefficient of $\eta^{m_{0}}$ in $P_{1}(\xi, \eta)$ does not vanish anywhere in $\Xi$ and that the $\lambda_{t}(\xi)$ are continuous in $\Xi$. 
and

$$
V_{s}=H_{s}(\xi, L u)
$$

Clearly, $\alpha_{s}(\xi)$ is continuous in $\Xi$ and homogeneous in $\xi$ of degree one. Combining (3.3) and (3.4) we have, in view of Corollary 3.1, that there is a constant $C$ such that

$$
\begin{aligned}
\sum \alpha_{s}(\xi)\left|\int J_{0}^{-1} \sum P_{k}\left(H_{s} S_{k}-P_{k} V_{s}\right) d \eta-\pi i\left(H_{s} u\right)^{\wedge}\right|^{2} \\
+\frac{1}{2} \int J_{0}^{-1} \Sigma\left|P_{k} S_{j}-P_{j} S_{k}\right|^{2} d \eta+\Sigma|\xi|\left|Q_{j} u\right|^{2} \\
\quad-\varepsilon \int J_{0}^{-1} \sum\left|P_{k} T-R S_{k}\right|^{2} d \eta \\
\leqq \int\left\{C \sum\left|\left(P_{k} u\right)^{\sim}\right|^{2}-\varepsilon|R u|^{2}\right\} d \eta+\Sigma|\xi|\left|\left(Q_{j} u\right)^{\wedge}\right|^{2}
\end{aligned}
$$

We now show that the left-hand side of (3.5) is non-negative for $\varepsilon$ sufficiently small. Writing it in the form

$$
I_{1}+I_{2}+I_{3}-\varepsilon I_{4}
$$

we note that each $I_{t}$ is of the form

$$
\sum_{i, j=0}^{m_{0}-1} M_{i j}(\xi) \omega_{i} \bar{\omega}_{j},
$$

where each $M_{i j}(\xi)$ is continuous in $\Xi$ and homogeneous in $\xi$ of degree $2 m_{0}+1$. It therefore suffices to prove there is an $\varepsilon>0$ such that

$$
I_{1}+I_{2}+I_{3} \geqq \varepsilon I_{4}
$$

on the compact set $\mathscr{S}$ of all $\xi$, $\omega$ such that $\xi \in \Xi$ and $\sum_{i=0}^{m_{0}-1}\left|\omega_{i}\right|^{2}=1$. This will certainly be the case if

$$
I_{1}+I_{2}+I_{3}
$$

does not vanish on $\mathscr{S}$.

The only way $I_{2}$ can vanish for a particular $\xi, \omega$ is if

$$
\frac{P_{k}}{P_{j}} \equiv \frac{S_{k}}{S_{j}}
$$

identically in $\eta$, for each $j, k$. Since the degree of $S_{k}$ in $\eta$ is one less than that of $P_{k}$, this means that there is a polynomial $S(\eta)$ of degree one less than $P_{\xi}(\eta)$ such that

$$
\frac{P_{k}}{S_{k}} \equiv \frac{P_{\xi}}{S}
$$

identically in $\eta$ for each $k$. In this case 


$$
\frac{\sum_{k} \bar{P}_{k}\left(H_{s} S_{k}-P_{k} V_{s}\right)}{J_{0}}=\frac{H_{s} S-P_{\xi} V_{s}}{P_{\xi}} .
$$

If

$$
P_{k}(\xi, \eta)=\sum_{s=0}^{m^{\prime}} a_{k s}(\xi) \eta^{s},
$$

then we see by Lemma 3.5 that the numerator on the left-hand side of (3.7) is a polynomial in $\eta$ of degree $2 m_{0}-1$ with leading coefficient equal to

$$
\left(H_{s} u\right)^{\wedge} \sum\left|a_{k m_{0}}(\xi)\right|^{2}
$$

while the denominator is of degree $2 m_{0}$ and has leading coefficient equal to

$$
\sum_{k}\left|a_{k m_{0}}(\xi)\right|^{2} \neq 0
$$

Since the leading coefficient of $P_{\xi}$ is one, (3.7) tells us that $H_{s} S-P_{\xi} V_{s}$ is of degree one less than $P_{\xi}$ and has leading coefficient equal to $\left(H_{s} u\right)^{\wedge}$. Moreover, among the $H_{s}$ are the polynomials of the form

$$
N_{t}(\xi, \eta)=|\xi|^{m^{\prime}-t-m^{+}(\xi)-1} \eta^{t} P_{\xi}^{+}(\eta), \quad 0 \leqq t<m_{0}-m^{+}(\xi) .
$$

For such polynomials Lemma 3.6 tells us that

$$
\int \frac{H_{s} S-P_{\xi} V_{s}}{P_{\xi}} d \eta=-\pi i\left(H_{s} u\right)^{\wedge} .
$$

Hence, when $I_{2}=0$,

$$
\sum|\xi|\left|\left(N_{t} u\right)^{\wedge}\right|^{2} \leqq \text { const. } I_{1} .
$$

Thus the only way $I_{1}+I_{2}+I_{3}$ can vanish is if

$$
\begin{array}{rrr}
\left(N_{t} u\right)^{\wedge}=0, & 0 \leqq t<m_{0}-m^{+}(\xi), \\
\left(Q_{j} u\right)^{\wedge}=0, & 1 \leqq j \leqq m^{+}(\xi),
\end{array}
$$

where we assume that the first $\mathrm{m}^{+}(\xi)$ of the $Q_{j}$ are linearly independent modulo $P_{\xi}$. This, of course, can be accomplished by renumbering them if necessary. Since the $N_{t}$ are linearly independent multiples of $P_{\xi}^{+}$and the $Q_{j}$ are linearly independent modulo $P_{\xi}^{+}$, the set $\left\{N_{t}, Q_{j}\right\}$ is linearly independent (Lemma 3.7). Since there are $m_{0}$ of them and they are of degree less than $m_{0}$, the only way (3.9) and (3.10) can hold is if $\omega_{0}=\omega_{1}=\cdots=\omega_{m_{0}-1}=0$. But then $\xi, \omega$ is not in $\mathscr{S}$. Hence $I_{1}+I_{2}+I_{3}$ is positive in $\mathscr{S}$ and the proof is complete.

4. The proof of Theorem 2.1. We now return to the partial differential operators $R, P_{k}, Q_{j}$. The corresponding characteristic polynomials are not only polynomials in $\eta$ but also in the components of $\xi$. 
We now assume that the hypotheses (a)-(c) of Theorem 2.1 are satisfied and prove that (2.5) holds for all $u \in C_{0}^{\infty}\left(G \cup \partial_{1} G\right)$ and real $r$. By hypothesis (a) it is easily seen that the sphere $|\xi|=1$ is the union of a finite number of closed sets $\Xi_{i}$, each of which has property A relative to the $P_{k}(\xi, \eta)$. In fact, let $k$ be such that

$$
\sum_{|\mu|+m_{0}=m_{k}} a_{k \mu m_{0}} \xi^{\mu} \neq 0
$$

and let $V$ be the set of those $\xi$ on $|\xi|=1$ such that

$$
\sum_{|\mu|+m_{0}=m_{k}} a_{k \mu m_{0}} \xi^{\mu}=0
$$

Clearly $V$ is compact. Moreover, hypothesis (a) tells us that for each $\xi \in V$ there is a $q$ such that

$$
\sum_{|\mu|+m_{0}=m_{u}} a_{q \mu m_{0}} \xi^{\mu} \neq 0
$$

and hence (4.1) holds in some neighborhood of $\xi$. A finite covering of $V$ by such neighborhoods gives a suitable decomposition of $|\xi|=1$.

Next we note that (2.6) implies (3.2) for each $\Xi_{i}$. Moreover, if $P(\xi, \eta)$ is the characteristic polynomial of a constant coefficient operator $P$, it is easily checked by integration by parts that the Fourier transform of $P u$ with respect to all $n$ variables is precisely the operator $(P u)^{\sim}$ of $\S 3$. Likewise, the tangential Fourier transform of $P u$ is $(P u)^{\wedge}$ as defined there (cf. [12]).

Hence, by Theorem 3.1, there are constants $C_{i}$ such that

$$
\int\left|(R u)^{\sim}\right|^{2} d \eta \leqq C_{i}\left\{\sum_{k}|\xi|^{2\left(m-m_{k}\right)} \int\left|\left(P_{k} u\right)^{\sim}\right|^{2} d \eta+\sum_{j}|\xi|^{2\left(m-v_{j}\right)-1}\left|\left(Q_{j} u\right)^{\wedge}\right|^{2}\right\}
$$

for all $u \in C_{0}^{\infty}\left(G \cup \partial_{1} G\right)$ and $\xi$ such that $\xi /|\xi| \in \Xi_{i}$. Summing over $i$, we have that

$$
\int\left|(R u)^{\sim}\right|^{2} d \eta \leqq C\left\{\sum_{k}|\xi|^{2\left(m-m_{k}\right)} \int\left|\left(P_{k} u\right)^{\sim}\right|^{2} d \eta+\sum_{j}|\xi|^{2\left(m-v_{j}\right)-1}\left|\left(Q_{j} u\right)^{\wedge}\right|^{2}\right\}
$$

for all $u \in C_{0}^{\infty}\left(G \cup \partial_{1} G\right)$ and $\xi \neq 0$. We now multiply both sides by $|\xi|^{2 r}$ and integrate with respect to $\xi$. This gives $(2.5)$ and the proof is complete.

5. Bounded domains. We now assume that $G$ is contained in the strip $0 \leqq x_{n} \leqq d$. We shall say that $\Xi$ has property B relative to a characteristic polynomial $P(\xi, \eta)$ if each root $\lambda(\xi)$ of $P(\xi, \eta)$ is single valued and continuous on $\Xi$ and satisfies either

(a) $\operatorname{Im} \lambda(\xi)>0$ at all interior points of $\Xi$, or

(b) $\operatorname{Im} \lambda(\xi) \leqq 0$ in $\Xi$.

If $\Xi$ has property B relative to $P$, we let $P^{s, t}(\xi, \eta)$ stand for the generic polynomial of the form 


$$
\frac{P(\xi, \eta)}{\prod_{q=1}^{s}\left(\eta-\alpha_{q}(\xi)\right) \prod_{q=1}^{t}\left(\eta-\beta_{q}(\xi)\right)}
$$

where the $\alpha_{q}(\xi)$ satisfy (a) and the $\beta_{q}(\xi)$ satisfy (b). If either $s$ or $t$ is negative, we set $P^{s, t}(\xi, \eta) \equiv 0$. We retain the notation of $\S 3$ and state

THEOREM 5.1. For any set of non-negative integers $\rho,\left\{l_{k}\right\}$ satisfying $\rho \leqq \max l_{k}$, there is a constant $C$ such that

$$
\begin{aligned}
\int\left|(R u)^{\sim}\right|^{2} d \eta \leqq & C\left\{\sum_{k}|\xi|^{2\left(m-m_{k}+l_{k}\right)} \int\left|\left(P_{k} u\right)^{\sim}\right|^{2} d \eta\right. \\
& \left.+\sum_{j}|\xi|^{2\left(m-v_{j}\right)-1}\left(1+\sum_{s=0}^{\rho}|\xi|^{2 s+1}\right)\left|\left(Q_{j} u\right)^{\wedge}\right|^{2}\right\}
\end{aligned}
$$

for all $u \in C_{0}^{\infty}\left(G \cup \partial_{1} G\right)$ and $\xi$ such that $\xi /|\xi| \in \Xi$, if

(a) $\Xi$ has property $\mathrm{B}$ relative to each $P_{k}(\xi, \eta)$ and property $\mathrm{A}$ relative to the polynomials $P_{k}^{0, l_{k}}(\xi, \eta)$;

(b) there are non-negative integers $\rho_{k} \leqq \min \left(\rho+1, l_{k}\right)$ such that

$$
|R(\xi, \eta)|^{2} \leqq K \sum_{k} \sum_{s=0}^{\rho_{k}}|\xi|^{2\left(m-m_{k}+l_{k}\right)}\left|P_{k}^{s, l_{k}-s}(\xi, \eta)\right|^{2}
$$

for all real $\eta$ and $\xi \in \Xi$, where summation is taken over all polynomials of the form $P_{k}^{s, l_{k}-s}(\xi, \eta)$;

(c) the $Q_{j}(\xi, \eta)$ cover the polynomials $P_{k}^{0, \sigma_{k}}(\xi, \eta)$, where

$$
\sigma_{k}=\min \left(l_{k}-\rho_{k}, l_{k}-\rho\right) \text {. }
$$

We shall prove Theorem 5.1 at the end of this section. We first derive some consequences. Setting $\rho$ and the $\rho_{k}$ equal to zero in Theorem 5.1 we have

COROLlaRY 5.1. For any non-negative integers $l_{k}$ there is a constant $C$ such that

$$
\begin{aligned}
& \int\left|(R u)^{\sim}\right|^{2} d \eta \\
& \quad \leqq C\left\{\sum_{k}|\xi|^{2\left(m-m_{k}+l_{k}\right)} \int\left|\left(P_{k} u\right)^{\sim}\right|^{2} d \eta+\sum_{j}(1+|\xi|)|\xi|^{2\left(m-v_{j}\right)-1}\left|\left(Q_{j} u\right)^{\wedge}\right|^{2}\right\}
\end{aligned}
$$

for all $u \in C_{0}^{\infty}\left(G \cup \partial_{1} G\right)$ and $\xi$ such that $\xi /|\xi| \in \Xi$, if

(a) $\Xi$ has property $\mathrm{B}$ relative to each $P_{k}(\xi, \eta)$ and property $\mathrm{A}$ relative to the polynomials $P_{k}^{0, l_{k}}(\xi, \eta)$;

(b) there is a constant $K$ such that

$$
|R(\xi, \eta)|^{2} \leqq K \sum_{k}|\xi|^{2\left(m-m_{k}+l_{k}\right)}\left|P_{k}^{0} l_{k}(\xi, \eta)\right|^{2}
$$

for real $\eta$ and $\xi \in \Xi$;

(c) the $Q_{j}(\xi, \eta)$ cover the polynomials $P_{k}^{0, l_{k}}(\xi, \eta)$. 
REMARK 5.1. If we take all the $l_{k}$ to be zero in Corollary 5.1 we obtain

$$
\begin{aligned}
& \int|R u|^{2} d \eta \\
& \quad \leqq C\left\{\sum_{k}|\xi|^{2\left(m-m_{k}\right)} \int\left|P_{k} u\right|^{2} d \eta+\sum_{j}(1+|\xi|)|\xi|^{2\left(m-v_{j}\right)-1}\left|Q_{j} u\right|^{2}\right\},
\end{aligned}
$$

which is slightly weaker than inequality (3.1).

COROLlaRY 5.2. Inequality (5.1) holds for non-negative integers $\rho$ and $l_{k}$ such that $\rho \leqq \max l_{k}$, if

(a) $\Xi$ has property $\mathrm{B}$ relative to each $P_{k}(\xi, \eta)$ and property $\mathrm{A}$ relative to the $P_{k}^{0, l_{k}}(\xi, \eta)$

(b) there is a constant $K$ such that

$$
|R(\xi, \eta)|^{2} \leqq K \sum_{k}|\xi|^{2\left(m-m_{k}+l_{k}\right)} \sum_{t=0}^{\rho+1}\left|P_{k}^{t, l_{k}-t}(\xi, \eta)\right|^{2}
$$

for real $\eta$ and $\xi \in \Xi$;

(c) the $Q_{j}(\xi, \eta)$ cover the polynomials $P_{k}^{0 . l_{k}-\rho}(\xi, \eta)$.

Proof. Take $\rho_{k}=\min \left(\rho+1, l_{k}\right)$ in Theorem 5.1. Clearly (5.4) implies (5.2). Moreover, since $l_{k}-\rho \geqq \sigma_{k}$, if the $Q_{j}$ cover the $P_{k}^{0, \sigma_{k}}$, they surely cover the $P_{k}^{0, l_{k}-\rho}$.

Corollary 5.3. Assume that $m_{1}=m+1$. Then

$$
\int\left|(R u)^{\sim}\right|^{2} d \eta \leqq C\left\{\int\left|\left(P_{1} u\right)^{\sim}\right|^{2} d \eta+\sum_{j}(1+|\xi|)|\xi|^{2\left(m-v_{j}\right)-1}\left|\left(Q_{j} u\right)^{\wedge}\right|^{2}\right\}
$$

if $\Xi$ has properties A and $\mathrm{B}$ relative to $P_{1}(\xi, \eta)$,

$$
|R(\xi, \eta)|^{2} \leqq K \sum\left(\left|P_{1}^{0,1}(\xi, \eta)\right|^{2}+\left|P_{1}^{1,0}(\xi, \eta)\right|^{2}\right),
$$

and the $Q_{j}$ cover the $P_{1}^{0,1}(\xi, \eta)$.

In certain applications it is convenient to take some of the $P_{k}(\xi, \eta)$ to be the same. For instance we have

THEOREM 5.2. For any set of non-negative integers $\rho,\left\{l_{k}\right\}$ with $\rho \leqq \max l_{k}$, there is a constant $C$ such that

$$
\begin{aligned}
\int|(R u) \sim|^{2} d \eta \leqq C\left\{\sum_{k} \sum_{s=0}^{l_{k}}|\xi|^{2\left(m-m_{k}+s\right)} \int\left|\left(P_{k} u\right)^{\sim}\right|^{2} d \eta\right. \\
\left.+\sum_{j}|\xi|^{2\left(m-v_{j}\right)-1}\left(1+\sum_{s=0}^{\rho}|\xi|^{2 s+1}\right)\left|\left(Q_{j} u\right)^{\wedge}\right|^{2}\right\}
\end{aligned}
$$

for all $u \in C_{0}^{\infty}\left(G \cup \partial_{1} G\right)$ and $\xi$ satisfying $\xi /|\xi| \in \Xi$, if

(a) $\Xi$ has property A relative to the $P_{k}(\xi, \eta)$ and property $\mathrm{B}$ relative to each $P_{k}(\xi, \eta)$; 
(b) there are non-negative integers $\rho_{k} \leqq \min \left(\rho+1, l_{k}\right)$ such that

$$
|R(\xi, \eta)|^{2} \leqq K \sum_{k} \sum_{s=0}^{\rho_{k}} \sum_{t=0}^{l_{k}-s}|\xi|^{2\left(m-m_{k}+s+t\right)}\left|P_{k}^{s, t}(\xi, \eta)\right|^{2}
$$

for real $\eta$ and $\xi \in \Xi$;

(c) the $Q_{j}(\xi, \eta)$ cover the $P_{k}^{0, \sigma_{k}}(\xi, \eta), \sigma_{k}=\min \left(l_{k}-\rho_{k}, l_{k}-\rho\right)$.

Proof. Set $P_{k s}(\xi, \eta)=P_{k}(\xi, \eta), l_{k s}=s, \rho_{k s}=\rho_{k}, s=0,1, \cdots, l_{k}$, and apply Theorem 5.1. One sees immediately that hypotheses (a) and (b) are satisfied. As for (c), we note that the $Q_{j}$ cover the polynomials $P_{k}^{0, \sigma_{k k}}$ where

$$
\sigma_{k s}=\min \left(l_{k s}-\rho_{k s}, l_{k s}-\rho\right) \text {. }
$$

Hence they cover the larger set $P_{k}^{0, \sigma_{k} s}$ (cf. Lemma 5.4 below), and the proof is complete.

The proof of Theorem 5.1 will be based upon the following lemmas.

LEMMA 5.1. For $t \geqq 0$ and $s \geqq 1$ there is a constant $C$ depending on $d$ such that for any characteristic polynomial $P(\xi, \eta)$

$$
\begin{aligned}
\int\left|\left(P^{0, t} u\right)^{\sim}\right|^{2} d \eta & \leqq C \int\left|(P u)^{\sim}\right|^{2} d \eta, \\
\left|\left(P^{0, t} u\right)^{\wedge}\right|^{2} & \leqq C \int\left|(P u)^{\sim}\right|^{2} d \eta, \\
\int\left|\left(P^{s, t} u\right)^{\sim}\right|^{2} d \eta & \leqq C\left\{\int\left|(P u)^{\sim}\right|^{2} d \eta+\sum_{q=1}^{s}\left|\left(P^{q, t} u\right)^{\wedge}\right|^{2}\right\}
\end{aligned}
$$

for all $u \in C_{0}^{\infty}\left(G \cup \partial_{1} G\right)$ and $\xi$ such that $\xi /|\xi| \in \Xi$.

Proof. The lemma follows from Parseval's identity and repeated applications of the inequalities

$$
\begin{array}{ll}
|g(t)| \leqq C \int_{0}^{d}|(D-i \lambda) g(\tau)| d \tau, & \operatorname{Im} \lambda \leqq 0, \\
|g(t)| \leqq C\left(\int_{0}^{d}|(D-i \lambda) g(\tau)| d \tau+|g(0)|\right), & \operatorname{Im} \lambda>0,
\end{array}
$$

where $D$ denotes differentiation with respect to $\tau$. The inequalities (5.10) and (5.11) hold for differentiable functions $g(t)$ in $0 \leqq t \leqq d$ which vanish for $t=d$. They are well known and easily proved (cf., e.g., [14]).

LEMMA 5.2. If

$$
P(\xi, \eta)=\eta-\beta(\xi)
$$

is a characteristic polynomial, then

$$
|\operatorname{Im} \beta|^{2} \int|\tilde{u}|^{2} d \eta \leqq \int\left|(P u)^{\sim}\right|^{2} d \eta+2 \pi \operatorname{Im} \beta|\hat{u}|^{2}
$$

for all $u \in C_{0}^{\infty}\left(G \cup \partial_{1} G\right)$. $G$ need not be bounded for (5.12) to hold. 
Proof. We have

$$
\begin{aligned}
\left|(P u)^{\sim}\right|^{2}= & |(\eta-\beta) \tilde{u}+\hat{u}|^{2}=|\eta-\beta|^{2}|\tilde{u}|^{2}+2 \operatorname{Re}(\eta-\beta) \tilde{u} \hat{u}^{-}+|\hat{u}|^{2} \\
= & {\left[\eta^{2}-2 \operatorname{Re} \beta \eta+(\operatorname{Re} \beta)^{2}+(\operatorname{Im} \beta)^{2}\right]|\tilde{u}|^{2}+2 \operatorname{Re}(\eta-\beta) \tilde{u} \hat{u}^{-}+|\hat{u}|^{2} } \\
= & (\eta-\operatorname{Re} \beta)^{2}|\tilde{u}|^{2}+2 \operatorname{Re}(\eta-\operatorname{Re} \beta) \tilde{u} \hat{u}^{-}+|\hat{u}|^{2}+(\operatorname{Im} \beta)^{2}|\tilde{u}|^{2} \\
& +2 \operatorname{Re}(-i \operatorname{Im} \beta) \tilde{u} \hat{u}^{-}=|(\eta-\operatorname{Re} \beta) \tilde{u}+\hat{u}|^{2}+(\operatorname{Im} \beta)^{2}|\tilde{u}|^{2} \\
& +2 \operatorname{Re}(-i \operatorname{Im} \beta) \tilde{u} \hat{u}^{-} .
\end{aligned}
$$

Hence

$$
\begin{aligned}
\int\left|(P u)^{\sim}\right|^{2} d \eta & \geqq(\operatorname{Im} \beta)^{2} \int|\tilde{u}|^{2} d \eta+2 \operatorname{Re}(-i \operatorname{Im} \beta) \hat{u}^{-} \int \tilde{u} d \eta \\
& =(\operatorname{Im} \beta)^{2} \int|\tilde{u}|^{2} d \eta-2 \pi \operatorname{Im} \beta|\hat{u}|^{2}
\end{aligned}
$$

by Lemma 3.4, and the proof is complete.

LemMa 5.3. Assume that $\beta(\xi)$ is a root of the characteristic polynomial $P(\xi, \eta)$ of multiplicity $>s$ and such that $\operatorname{Im} \beta(\xi) \leqq 0$ in $\Xi$. Set

$$
P_{s}^{\beta}(\xi, \eta)=\frac{P(\xi, \eta)}{(\eta-\beta(\xi))^{s}} .
$$

Then for $t \leqq s$

$$
|\operatorname{Im} \beta|^{2(t-1)}\left|\left(P_{s}^{\beta} u\right)^{\wedge}\right|^{2} \leqq C \int\left|(P u)^{\sim}\right|^{2} d \eta
$$

Proof. By repeated use of (5.12) we have, since $\operatorname{Im} \beta \leqq 0$,

$$
|\operatorname{Im} \beta|^{2(t-1)} \int\left|\left(P_{t-1}^{\beta} u\right)^{\sim}\right|^{2} d \eta \leqq \int\left|(P u)^{\sim}\right|^{2} d \eta
$$

We then apply (5.8) to complete the proof.

LEMMA 5.4. If the $Q_{j}$ cover a subset of the $P_{k}$, then they cover the whole set.

Proof. Trivial (cf. [12, p. 55]).

Proof of Theorem 5.1. Since each $\sigma_{k} \leqq l_{k}-\rho_{k}$, the $Q_{j}$ cover the polynomials $P_{k}^{0, l_{k}}(\xi, \eta)$ and hence, by Lemma 5.4 , the larger set $P_{k}^{s, l_{k}-s}(\xi, \eta), 0 \leqq s \leqq \rho_{k}$. Applying Theorem 3.1, we see, by hypothesis (b), that

$$
\int\left|(R u)^{\sim}\right|^{2} d \eta
$$

$$
\leqq C\left\{\sum_{k} \sum_{s=0}^{\rho_{k}}|\xi|^{2\left(m-m_{k}+l_{k}\right)} \int\left|\left(P_{k}^{s, l_{k}-s} u\right)^{\sim}\right|^{2} d \eta+\sum_{j}|\xi|^{2\left(m-v_{j}\right)-1}\left|Q_{j} u\right|^{2}\right\}
$$


Now by Lemma 5.1, for any particular $\kappa$

$$
\int\left|\left(P_{\kappa}^{0, l \kappa-s} u\right)^{\sim}\right|^{2} d \eta \leqq C \int\left|\left(P_{\kappa} u\right)^{\sim}\right|^{2} d \eta
$$

and

$$
\int\left|\left(P_{\kappa}^{s . l \kappa-s} u\right)^{\sim}\right|^{2} d \eta \leqq C\left\{\int\left|\left(P_{\kappa} u\right)^{\sim}\right|^{2} d \eta+\sum_{q=1}^{s}\left|\left(P_{\kappa}^{q, l \kappa-s} u\right)^{\wedge}\right|^{2}\right\}
$$

for $s \geqq 1$. We are going to show that

$$
\begin{aligned}
& |\xi|^{2\left(m-m_{\kappa}+l_{\kappa}\right)}\left|\left(P_{\kappa}^{q .} l^{\prime \kappa-s} u\right) \wedge\right|^{2} \\
& \leqq C\left\{\sum_{k}|\xi|^{2\left(m-m_{k}+l_{k}\right)}\left|\left(P_{k}^{0, l_{k}-s+q} u\right)^{\wedge}\right|^{2}\right. \\
& +\sum_{k, h} \sum_{\rho=l \kappa-s+q+1}^{\rho_{k h}}|\xi|^{2\left(m-m_{k}+l_{k}\right)}\left|\operatorname{Im} \beta_{k h}\right|^{2(\rho+s-l \kappa-q)}\left|\left(P_{\kappa \rho}^{\beta_{k h}} u\right)^{\wedge}\right|^{2} \\
& \left.+\sum_{j}|\xi|^{2\left(m+s-v_{j}-q\right)}\left|\left(Q_{j} u\right)^{\wedge}\right|^{2}\right\}
\end{aligned}
$$

for each $\kappa, s, q$ such that $1 \leqq q \leqq s \leqq \rho_{\kappa}$ where the $\beta_{k h}(\xi)$ are the roots of $P_{k}(\xi, \eta)$ which have nonpositive imaginary parts in $\Xi$ and are of multiplicity $\rho_{k h} \geqq l_{k}-s+q+1$. Moreover, it is to be understood that the term containing the $\beta_{k h}(\xi)$ in (5.17) is to appear only when $l_{k}-s+q+1 \geqq 2$.

Once (5.17) is proved, the theorem then follows from (5.14-5.17) and the fact that each term on the right-hand side of (5.17) can be estimated in terms of the right-hand side of (5.1). This is clear for the first term by (5.7) (recall that $P^{0, l_{k}-s+q} \equiv 0$ if $\left.l_{k}-s+q<0\right)$. For the middle term we employ Lemma 5.3, making use of the fact that this term comes into play only when $l_{k}-s+q \geqq 1$. As for the third term, it is obviously contained in the second term on the righthand side of (5.1).

It therefore remains only to prove (5.17). Each term in (5.17) is of the form

$$
\sum M_{i h}(\xi) \omega_{i} \bar{\omega}_{h},
$$

where the $M_{i h}(\xi)$ are continuous in $\xi$ and homogeneous of degree $m+s-q+2$. Following the proof of Theorem 3.1 we see that we must show that the vanishing of the right-hand side of (5.17) implies that $\omega_{0}=\omega_{1}=\cdots=\omega_{m_{0}^{s}, q_{-1}}=0$, when $m_{0}^{s, q}$ is the greatest normal order of the $P_{k}^{0, l_{k}-s+q}$. But this is indeed the case. For let $P_{\xi}(\eta)$ be the greatest common divisor of the $P_{k}^{0, l_{k}-s+q}$ and let $P_{\xi}^{+}(\eta)$ be its factor containing its roots with non-negative imaginary parts. Denote the normal order of $P_{\xi}^{+}(\eta)$ by $m^{+}(\xi)$. Since $l_{k}-s+q \geqq \sigma_{k}$, we see by hypothesis (c) that there are $m^{+}(\xi)$ of the $Q_{j}$ which are linearly independent modulo $P_{\xi}^{+}(\eta)$. Moreover, if at $\xi$ there are no common roots of the $P_{k}^{0, l_{k}-s+q}$ with negative imaginary parts and having multiplicities greater than one, then there are $m_{0}^{s, q}-m^{+}(\xi)$ polynomials among them which are linearly independent. Other- 
wise we adjoin to them the polynomials $P_{k \rho}^{\beta_{k h}}$ corresponding to such roots. It should be noticed that the factors $\operatorname{Im} \beta_{k h}$ accompanying the $P_{k \rho}^{\beta_{k h}}$ in (5.17) do not vanish for such roots. Thus we see that there are $m_{0}^{s, q}-m^{+}(\xi)$ polynomials among the $P_{k}^{0, l_{k}-s+q}$ and $P_{k \rho}^{\beta_{k h}}$ which are linearly independent. Since the latter all contain $P_{\xi}^{+}(\eta)$ as a factor, we have among the $P_{k}^{0, l_{k}-s+q}, P_{k s}^{\beta_{k h}}$, and $Q_{j}, m_{0}^{s, q}$ polynomials which are linearly independent (Lemma 3.7). Since they are all of normal order less than $m_{0}^{s, q}$ the simultaneous vanishing of the

$$
\left(P_{k}^{0, l_{k}-s+q} u\right)^{\wedge},\left(P_{k \rho}^{\beta_{k} h} u\right)^{\wedge},\left(Q_{j} u\right)^{\wedge}
$$

mplies that $\omega_{0}=\omega_{1}=\cdots=\omega_{m_{0}^{s, q}}^{s-1}=0$ and the proof is complete.

6. Further results and the remaining proofs. An immediate consequence of Theorem 5.1 is

THEOREM 6.1. For any set of non-negative integers $\rho,\left\{l_{k}\right\}$ with $\rho \leqq \max l_{k}$, there is a constant $C$ such that

$$
|R u|_{r-m} \leqq C\left\{\sum_{k}\left|P_{k} u\right|_{r-m_{k}+l_{k}}+\sum_{j} \sum_{s=0}^{\rho+1}\left\langle Q_{j} u\right\rangle_{r-v_{j}+s-1}\right\}
$$

for all real $r$ and $u \in C_{0}^{\infty}\left(G \cup \partial_{1} G\right)$, if the sphere $|\xi|=1$ is the union of a finite number of closed sets $\Xi_{i}$ on each of which hypotheses (a)-(c) of Theorem 5.1 hold.

The proof is obvious. By Theorem 5.1, inequality (5.1) holds for each $\Xi_{i}$. Summing over all the $\Xi_{i}$, multiplying by $|\xi|^{2(r-m)}$, employing the trivial inequality $2 \leqq|\xi|^{-1}+|\xi|$, and integrating with respect to $\xi$, we obtain (6.1).

RemarK 6.1. Statements similar to Theorem 6.1 hold in connection with Corollaries 5.1-5.3 and Theorem 6.2. For the latter we obtain the inequality

$$
|R u|_{r-m} \leqq C\left\{\sum_{k} \sum_{s=0}^{l_{k}}\left|P_{k} u\right|_{r-m_{k}+s}+\sum_{j} \sum_{s=0}^{\rho+1}\left\langle Q_{j} u\right\rangle_{r-v_{j}+s-1}\right\}
$$

when $|\xi|=1$ can be broken up into a finite number of closed sets on each of which hypotheses (a)-(c) of Theorem 5.2 hold.

When $G$ is bounded and $r$ is sufficiently large we can simplify (6.2) by employing an inequality due to Schwartz [13].

LEMMA 6.1. Let $D$ be a bounded domain in $E^{n-1}$ and $h$ a non-negative real number. Then there is a constant $C$ depending only upon $h$ and the size of $D$ such that

$$
\int|\xi|^{2 h}|\hat{\phi}|^{2} d \xi \leqq C \int_{|\xi|>1}|\xi|^{2 h}|\hat{\phi}|^{2} d \xi
$$

for all functions $\phi\left(x^{\prime}\right)$ with compact support in $D$.

For the proof of Lemma 6.1 we refer to Schwartz [13, Theorem 6.1]. Employing it we have 
THEOREM 6.2. Assume that $|\xi|=1$ is the union of a finite number of closed sets on each of which hypotheses (a)-(c) of Theorem 5.2 hold. Then if $G$ is bounded, there is a constant $C$ such that

$$
|R u|_{r-m} \leqq C\left\{\sum_{k}\left|P_{k} u\right|_{r-m_{k}+l_{k}}+\sum_{j}\left\langle Q_{j} u\right\rangle_{r-v_{j}+\rho}\right\}
$$

for all $u \in C_{0}^{\infty}\left(G \cup \partial_{1} G\right)$ and real $r \geqq \max _{j, k}\left(m_{k}, v_{j}+1\right)$.

Proof. We apply Lemma 6.1 to inequality (6.2). By assumption, the powers of $|\xi|$ in the integrals on the right-hand side of (6.2) are non-negative. Hence by Lemma 6.1 they are majorized by a constant times the same integrals over $|\xi|>1$. But on this set $|\xi|^{2\left(r-m_{k}+s\right)} \leqq|\xi|^{2\left(r-m_{k}+l_{k}\right)}$, and the proof is complete.

We now give the remaining proofs of the theorem of $\$ 2$.

Proof of Theorem 2.2. We first note that $|\xi|=1$ can be subdivided into a finite number of closed sets $\Xi_{i}$ each of which has property B relative to each $P_{k}(\xi, \eta)$. Next, by hypothesis (a) each $\Xi_{i}$ can be subdivided into a finite number of closed sets $\Xi_{i h}$ each of which has property A relative to the $P_{k}(\xi, \eta)$. Thus by Theorem 6.2, we need only show that hypotheses (b) and (c) of Theorem 5.2 are fulfilled in each $\Xi_{i h}$.

It is easily seen that (2.8) implies (5.6). In fact

$$
\left|\frac{\partial^{s} P_{k}(\xi, \eta)}{\partial \eta^{s}}\right| \leqq \text { const. } \sum_{t=0}^{s}\left|P_{k}^{t, s-t}(\xi, \eta)\right|^{2},
$$

and if $t \leqq s \leqq \rho_{k} \leqq l_{k}$, then $s-t \leqq l_{k}-t$. Thus the terms on the right-hand side of (6.5) are among the terms on the right-hand side of (5.6).

Finally, we note that for each $\xi$ and $k, P_{k}^{\left(\sigma_{k}\right)}(\xi, \eta)$ is the greatest common divisor for all polynomials of the form $P_{k}^{0, \sigma_{k}}(\xi, \eta)$. Hence the $Q_{j}$ cover the $P_{k}^{0, \sigma_{k}}(\xi, \eta)$ if and only if they cover the $P_{k}^{\left(\sigma_{k}\right)}(\xi, \eta)$. Thus the proof is complete.

Proof of Theorem 2.3. As before, we can prove the theorem by showing that hypotheses (b) and (c) of Theorem 5.2 are satisfied for any closed set $\Xi$ of $|\xi|=1$ which has property A relative to the $P_{k}(\xi, \eta)$ and property B relative to each $P_{k}(\xi, \eta)$. Thus we may assume that

for $\xi \in \Xi$. If

$$
\sum a_{1 \mu m_{0}} \xi^{\mu} \neq 0
$$

then $\theta>0$ and

$$
\theta=\min _{\xi \in \Xi}\left|\sum a_{1 \mu m_{0}} \xi^{\mu}\right|
$$

$$
\left|P_{1}(\xi, \eta)\right| \geqq \theta \eta^{m_{0}}-|E(\xi, \eta)|
$$

for $\xi \in \Xi$, where $E(\xi, \eta)$ is a polynomial in $\eta$ of degree less than $m_{0}$ with coefficients depending on $\xi$ which are uniformly bounded for $\xi \in \Xi$. Thus by (a') there are contants $K$ and $M$ such that 


$$
\frac{|R(\xi, \eta)|}{\left|P_{1}(\xi, \eta)\right|} \leqq K
$$

for $\xi \in \Xi$ and $|\eta|>M$. Moreover, by $\left(\mathrm{b}^{\prime}\right)$ the polynomials $P_{k}^{0, t}(\xi, \eta)$,

$$
0 \leqq t \leqq \gamma=\min \left(\rho+1, \min l_{k}\right)
$$

cannot all vanish for any real $\xi, \eta$ such that $|\xi|=1$. Taking each $\rho_{k}=\gamma$, we see that there is a constant $K$ such that (5.6) holds on the compact set $\xi \in \Xi,-M \leqq \eta \leqq M$. Combining this with (6.6) we see that (5.6) holds for all real $\xi, \eta$ such that $\xi \in \Xi$.

Finally, since $\sigma_{k} \leqq l_{k}-\rho$, we see that the $Q_{j}$ cover the $P_{k}^{0, l_{k}-\rho}(\xi, \eta)$ and hence the $P_{k}^{\left(l_{k}-s\right)}(\xi, \eta)$. This completes the proof.

Proof of Theorem 2.4. Here we apply Corollary 5.1 (cf. Remark 6.1). We note that in two dimensions the whole set $|\xi|=1$ (i.e., $\xi= \pm 1$ ) has properties $A$ and $B$ relative to any and all polynomials. Moreover,

$$
\left|\frac{\partial^{s+t} P_{k}(\xi, \eta)}{\partial \xi^{s} \partial \eta^{t}}\right|^{2} \leqq \text { const. } \Sigma\left|P_{k}^{0, s+t}(\xi, \eta)\right|^{2}
$$

and hence (2.10) implies (5.3). Finally, we observe, as before, that $P^{(h)}(\xi, \eta)$ is the greatest common divisor of the polynomials $P^{0, h}(\xi, \eta)$. Thus hypothesis (c) of Corollary 5.1 is also satisfied and the proof is complete.

The proof of Theorem 2.5 relies upon the following fact

Lemma 6.2. If $R(\xi, \eta)$ and the $P_{k}(\xi, \eta)$ are homogeneous, each $m_{k} \geqq m$, and $R(\xi, \eta)$ is weaker than the $P_{k}(\xi, \eta)$, then there is a constant $C$ such that

$$
|R(\xi, \eta)|^{2} \leqq C \sum_{s+t=m_{k}-m}\left|\frac{\partial^{s+t} P_{k}(\xi, \eta)}{\partial \xi^{s} \partial \eta^{t}}\right|^{2}
$$

for all real $\xi, \eta$.

Proof. Let $m_{0}$ be the maximum normal order of the $P_{k}(\xi, \eta)$ and $m_{0}^{\prime}$ the normal order of $R(\xi, \eta)$. Then by (2.11), $m_{0} \geqq m_{0}^{\prime}$. Assume that $P_{1}(\xi, \eta)$ is of normal order $m_{0}$. Then there is a derivative of $P_{1}(\xi, \eta)$ of order $m_{1}-m$ which is of degree $\geqq m_{0}^{\prime}$ in $\eta$. If $m_{0} \geqq m$, it is

$$
\frac{\partial^{m_{1}-m} P_{1}(\xi, \eta)}{\partial \xi^{m_{1}-m_{0}} \partial \eta^{m_{0}-m}}
$$

and if $m_{0}<m$, it is

$$
\frac{\partial^{m_{1}-m} P_{1}(\xi, \eta)}{\partial \xi^{m_{1}-m}} .
$$

Thus as any event, there are constants $C$ and $M$ such that (6.7) holds for $\xi= \pm 1$ and $|\eta|>M$. 
Now the only way (6.7) can fail to hold on the compact set $\xi= \pm 1,|\eta| \leqq M$ is when all the derivatives on the right-hand side have a real root $\alpha$ with multiplicity $\geqq h \geqq 1$ and $\alpha$ is either not a root of $R(\xi, \eta)$ or a root with multiplicity less than $h$. Assume this to be the case. Since the $P_{k}(\xi, \eta)$ are homogeneous, Euler's formula shows us that $\alpha$ is a root of all derivatives of $P_{k}(\xi, \eta)$ of order $\leqq m_{k}-m$. Hence $\alpha$ is a root of $P_{k}(\xi, \eta)$ with multiplicity at least $m_{k}-m+h$. Thus any derivative of $P_{k}(\xi, \eta)$ of order $p \leqq m_{k}-m+h$ has $\alpha$ as a root with multiplicity $m_{k}-m+h-p$. The degree in $\eta$ of such a derivative is, of course, $m_{k}-p$.

Now set $\xi_{N}=N, \eta_{N}=\alpha N+1$. Then $\eta_{N}-\alpha \xi=1$, while $\left|\eta_{N}-\beta \xi_{N}\right| \rightarrow \infty$ as $N \rightarrow \infty$ if $\beta \neq \alpha$. By the considerations above, we see that

$$
\frac{\partial^{s+t} P_{k}\left(\xi_{N}, \eta_{N}\right)}{\partial^{s} \xi \partial^{t} \eta}=O\left(N^{m-h}\right)
$$

for all $s$ and $t$. Since $\alpha$ is not a root of $R(\xi, \eta)$ with multiplicity $\geqq h$, we know that $\left|R\left(\xi_{N}, \eta_{N}\right)\right| / N^{m-h} \rightarrow \infty$, and hence (2.11) cannot hold. Thus our assumption is false and (6.7) must hold on the set $\xi= \pm 1$ and $|\eta| \leqq M$. Hence it holds for $|\xi|=1$ and all real $\eta$. By homogeneity, it holds for all real $\xi, \eta$, and the proof is complete.

Proof of Theorem 2.5. We set $\tau=r-m, l_{k}=m_{k}-m$ in Theorem 2.4. By Lemma 6.2, (6.7) holds. Moreover the right-hand side of (6.7) is majorized by

$$
\text { const. } \Sigma\left|P_{k}^{0, m_{k}-m}(\xi, \eta)\right|^{2} \text {. }
$$

The theorem now follows from Corollary 5.1 and Remark 6.1.

Added in Proof. The methods of the present paper can be adapted to give corresponding inequalities for operators which are not necessarily homogeneous. These inequalities permit one to obtain regularity up to the boundary for solutions of hypoelliptic boundary problems. Details will appear elsewhere.

\section{BIBLIOGRAPHY}

1. Shmuel Agmon, The coerciveness problem for integro-differential forms, J. Analyse Math. 6 (1958), 183-223.

2. Shmuel Agmon, Avron Douglis and Louis Nirenberg, Estimates near the boundary for solutions of elliptic partial differential equations satisfying general boundary conditions. I, Comm. Pure Appl. Math. 12 (1959), 623-727.

3. Nachman Aronszajn, On coercive integro-differential forms, Proceedings of the Conference on Partial Differential Equations, University of Kansas, Tech. Rep. No. 14, Lawrence, Kansas, 1954, pp. 94-106.

4. F. E. Browder, On regularity properties of solutions of elliptic differential equations, Comm. Pure Appl. Math. 9 (1956), 351-361.

5. - A priori estimates for solutions of elliptic boundary value problems. I, II, Proc. Nederl. Akad. Wetensch. 22 (1960), 145-169; 23 (1961), 404-410.

6. Lars Gårding, Dirichlet's problem for linear elliptic partial differential equations, Math. Scand. 1 (1953), 57-72. 
7. O. V. Guseva, On boundary problems for strongly elliptic systems, Dokl. Akad. Nauk SSSR 102 (1955), 1069-1072. (Russian)

8. Lars Hörmander, On the theory of general partial differential operators, Acta Math. 94 (1955), 161-284.

9. Bernard Malgrange, Existence et approximation des solutions des équations aux dérivées partielles et des équations de convolution, Ann. Inst. Fourier (Grenoble) 6 (1955-1956), 271-335.

10. Louis Nirenberg, Remarks on strongly elliptic partial differential equations, Comm. Pure Appl. Math. 8 (1955), 649-675.

11. Martin Schechter, On estimating elliptic partial differential operators in the $L_{2}$ norm Amer. J. Math. 79 (1957), 431-443.

12. - Integral inequalities for partial differential operators and functions satisfying general boundary conditions, Comm. Pure Appl. Math. 12 (1959), 37-66.

13. Laurent Schwartz, Su alcuni problemi della teoria della equazioni differenziali lineari di tipo ellittico, Rend. Sem. Mat. Fis. Milano 27 (1958), 1-41.

14. Vidar Thomée, Locally cogent boundary operators, Math. Scand. 7 (1959), 5-32.

15. François Trèves, Relations de domination entre opérateurs différentiels, Acta Math. 101 (1959), 1-139.

NeW YoRK UNIVERSITY,

New York, New YorK 